\title{
Wir brauchen einen kommunalen Rettungsfonds, aber nicht für alle Kommunen
}

Jörg Bogumil

Die kommunale Finanzkrise ist wahrlich kein neues Thema, aber infolge der Finanzkrise und einbrechender Gewerbe- und Einkommensteuereinnahmen - verschärft durch die Steuerpolitik der schwarz-gelben Bundesregierung - erhält sie eine neue Dimension, vor allem auch in der Wahrnehmung der Öffentlichkeit. Nach ausführlichen Berichten in „Focus“, „Spiegel“ und „Zeit“ betonen im Vorfeld der Landtagswahl auf einmal auch die Wahlkämpfer in Nordrhein-Westfalen (NRW), dass den Kommunen nun endlich geholfen werden muss. Allerdings leidet die Debatte nicht nur an mangelnder Glaubwürdigkeit der Parteien, die in der großen Koalition die Chance auf eine Gemeindefinanzreform ausgelassen oder im Bundesrat Ende letzten Jahres den Steuerreformbeschlüssen der Bundesregierung zugestimmt haben. Es fehlt auch an einer differenzierten Diskussion hinsichtlich des Ausmaßes und der Ursachen der kommunalen Finanzkrise und der notwendigen Handlungsschritte.

Lars Holtkamp beziffert in seiner in Kürze erscheinenden Studie für 2008-2010 ein Minus von 20,8 \% bei der Gewerbesteuer, ein Minus von 16,4 \% bei der Einkommensteuer und um 7,8 \% steigende Sozialausgaben (die Folgen einer möglichen weiteren Steuerreform, wie von der Bundesregierung angekündigt, sowie die Folgen des Bundesverfassungsgerichtsurteils zu Hartz IV noch nicht eingerechnet). Zwar treffen der Einbruch der Steuereinnahmen und der Anstieg der Sozialausgaben alle Kommunen, aber in höchst unterschiedlichem Ausmaß. Den „freien Fall der Kommunalfinanzen“, auf den der Städtetag im aktuellen Gemeindefinanzbericht aufmerksam macht, gibt es in vielen Kommunen schon seit Längerem. Deutlich wird dies, wenn man sich die Verschuldungssituation in den Kommunen anschaut. So liegen die Kassenkredite der Kommunen in Rheinland-Pfalz im Jahr 2007 bei $811 €$ pro Einwohner, in NRW bei $763 €$, gefolgt von Niedersachsen (521 €) und Hessen $(515 €)$. In den ostdeutschen Flächenländern sind es dagegen „nur" $190 €$ und in Bayern und Baden-Württemberg sogar nur $16 €$ bzw. $9 €$. Dass die hochverschuldeten Städte in NRW angesichts einer vierfach höheren Verschuldung als die ostdeutschen Flächenländer weiterhin jedes Jahr große Summen in den „Fond deutsche Einheit“ einbezahlen müssen, ist schon seit Langem nicht mehr zu rechtfertigen. Die Summe der in den letzten 20 Jahren in den Osten transferierten Gelder macht in manchen Kommunen des Ruhrgebietes ca. 30 \% ihrer Kassenkredite aus.

Aber auch in NRW trifft die kommunale Finanzkrise nicht alle Kommunen. Im Jahr 2008 mussten Städte wie Düsseldorf und Münster keine Kassenkredite aufnehmen, während der negative Spitzenreiter Oberhausen auf eine Summe von $5.411 €$ pro Einwohner kam, gefolgt von Hagen mit $4.123 €$ und Remscheid mit $3.616 €$. Die Steuereinnahmen in Düsseldorf liegen pro Einwohner dreimal so hoch wie in Oberhausen. Deutlich wird eines: Wenn man die jeweilige Ausgangssituation der Städte betrachtet, dann gibt es nicht die kommunale Finanzkrise, sondern diese schlägt vor allen in den Kommunen zu, die seit fast 20 Jahren mit hoher Erwerbslosigkeit, hohen Sozialausgaben und niedrigeren Steuereinnahmen zu kämpfen haben. Oberhausen, eigentlich das gesamte Ruhrgebiet, Städte wie Kaiserslautern, Cuxhaven, Wuppertal, Solingen oder Remscheid können sich nicht mehr selbstständig entschulden. Die gesamten Steuereinnahmen von Oberhausen aus zehn Jahren würden benötigt, um die Kassenkredite abzulösen. Für diese Städte, nicht für alle Kommunen, brauchen wir einen Rettungsfonds, finanziert durch Bund und Länder. Mehr wird angesichts der Haushaltslagen von Bund und Ländern auch nicht möglich sein. Wir brauchen vor allem kein Gießkannenprinzip, bei dem alle etwas erhalten, was aber den wirklich betroffenen Kommunen nichts nützt. Neben diesem Rettungsfonds ist zudem weiterhin über eine neue Gemeindefinanzstruktur nachzudenken, die konjunkturunabhängiger ausgestaltet ist. Dazu müssten sich aber auch die kommunalen Spitzenverbände untereinander einig werden.

Die Ursachen der kommunalen Haushaltskrise liegen aber nicht nur in diesen strukturellen, von den Kommunen oftmals wenig zu beeinflussenden Faktoren. Es gibt deutliche Unterschiede zwischen Kommunen mit annähernd gleichen strukturellen Rahmenbedingungen und viele Beispiele für kommunale Misswirtschaft von Crossborder-Geschäften über Spekulationsverluste hin zu kostenträchtigen Prestigeobjekten mit enormen Folgenkosten über Jahre. Deshalb muss ein Rettungsfonds für die wirklich überschuldeten Kommunen mit klaren Auflagen zur Kostenreduzierung verbunden werden. Selbstverschuldete Haushaltskrisen können nicht von außen aufgefangen werden, das wäre ein völlig falsches Anreizsignal. Möglichkeiten interner Einsparmaßnahmen, und zwar über die hinaus, die bereits auch aufgrund des Druckes der Aufsichtsbehörden oder durch durchsetzungsstarke Bürgermeister erfolgt sind, bestehen in verstärkter interkommunaler Kooperation z.B. in der Kulturpolitik oder Teilen der kommunalen Infrastruktur. Wenn dies freiwillig nicht gelingt, wie es angesichts der Kirchturmspolitik häufig der Fall ist, muss man von staatlicher Seite etwas nachhelfen.

Jörg Bogumil, Prof. Dr., Ruhr-Universität Bochum, Fakultät für Sozialwissenschaft, Lehrstuhl Öffentliche Verwaltung, Stadt- und Regionalpolitik. Arbeitsschwerpunkte: Modernisierung des öffentlichen Sektors, Stadt- und Regionalpolitik. e-mail: joerg.bogumil@ruhr-uni-bochum.de 